\title{
A new analytic representation of the ringdown waveform of coalescing spinning black hole binaries
}

\author{
Thibault Damour and Alessandro Nagar \\ Institut des Hautes Etudes Scientifiques, 91440 Bures-sur-Yvette, France
}

(Dated: October 15, 2018)

\begin{abstract}
We propose a new way of analyzing, and analytically representing, the ringdown part of the gravitational wave signal emitted by coalescing black hole binaries. By contrast with the usual linear decomposition of the multipolar complex waveform $h(t)$ in a sum of quasi-normal modes, our procedure relies on a multiplicative decomposition of $h(t)$ as the product of the fundamental quasi-normal mode with a remaining time-dependent complex factor whose amplitude and phase are separately fitted. As an illustrative example, we apply our analysis and fitting procedure to the ringdown part of a sample of sixteen $\ell=m=2$ equal-mass, spinning, nonprecessing, numerical waveforms computed with the $\mathrm{SP}_{\mathrm{E}} \mathrm{C}$ code, now publicly available in the SXS catalogue. Our approach yields an efficient and accurate way to represent the ringdown waveform, thereby offering a new way to complete the analytical effective-one-body inspiral-plus-plunge waveform.
\end{abstract}

\section{INTRODUCTION}

The numerical-relativity (NR) completion of the effective-one-body (EOB) approach [1-7] (usually called EOBNR) is a NR-informed analytical method that aims at giving an accurate modelization of the gravitational dynamics and waveforms of coalescing relativistic binaries (i.e., black holes and neutron stars) [8 22]. The EOB waveform for coalescing black-hole binaries is essentially made of the juxtaposition of two distinct parts: the inspiral-plus-plunge (or "insplunge") part (up to merger), and the subsequent ringdown part (after merger). The insplunge waveform is analytically defined by applying a sophisticated resummation procedure $6,23,24$ to the post-Newtonian-expanded waveform and dynamics 25]. At merger, the insplunge waveform is matched to the ringdown part, defined, up to now, as a linear superposition of quasi-normal modes (QNMs) of the final black hole. The standard approach (initiated in Ref. [2]) to compute this ringdown part is: (i) to identify the mass $M_{\mathrm{BH}}$ and angular momentum $J_{\mathrm{BH}}$ of the final black hole (either using the prediction of the EOB dynamics or using NR fitting formulas for these quantities [26, 27]); (ii) to use $\left(M_{\mathrm{BH}}, J_{\mathrm{BH}}\right)$ to compute a set of QNM frequencies [28, 29]; and (iii) to build a linear superposition of QNMs with coefficients determined by imposing some matching (i.e., continuity) conditions between such a ringdown waveform and the EOB insplunge waveform at (iv) a certain merger moment $t=t_{0}$ determined from the EOB dynamics (e.g., as the peak of the EOB orbital frequency). Such a procedure was found to work at a satisfactory level of robustness and accuracy in the nonspinning case (when complemented with some additional procedures, such as a matching over an interval around the time $t_{0}$ ) 10, 16, 21, 23]. However, the spinning case (as well as the case of higher modes in the nonspinning case 15]) proved to be more challenging because the gravitational wave frequency of a matched QNM-ringdown signal was found to rise too quickly after merger [16, 18]. To overcome this difficulty, Refs. [15, 16, 18] proposed

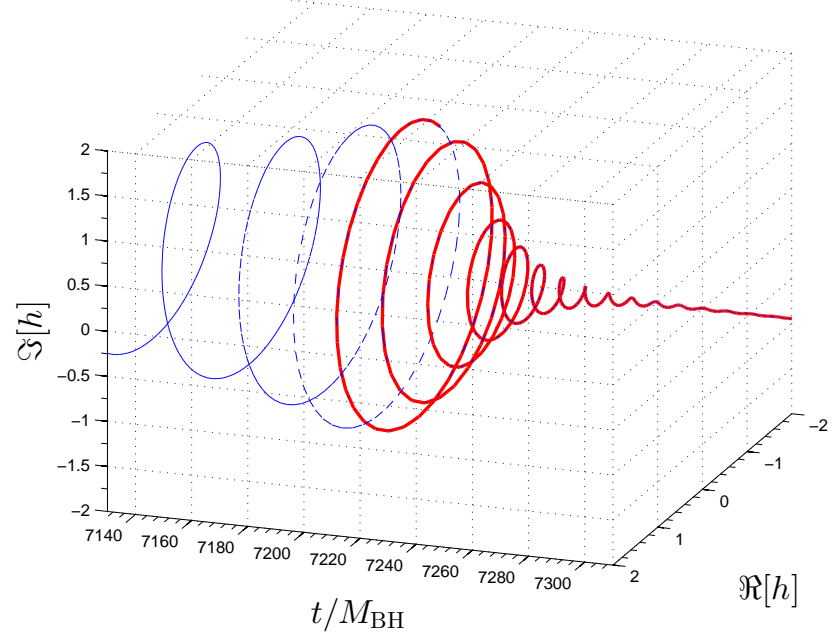

FIG. 1. Three-dimensional representation of the time evolution of the $\nu$-rescaled complex strain (metric) waveform $h \equiv h_{22} / \nu$, around merger, for dimensionless spin $\chi=0.97$. The thicker portion of the curve (red online) highlights the ringdown part.

to augment the analytical ringdown signal by including, besides the real QNMs modes, some pseudo-quasi-normal modes, i.e., fictitious modes with frequencies phenomenologically chosen so as to bridge the gap between the final gravitational wave frequency of the insplunge EOB waveform measured at merger and the frequency of the fundamental QNM.

In this paper, we propose a new, alternative, NRinformed strategy for constructing accurate analytical representations of the ringdown waveform of coalescing, spinning, black hole binaries. Here we shall consider only nonprecessing, equal-mass and equal-spin binaries, but our method is general, being based on a new way of analyzing and fitting the ringdown signal provided by NR simulations. In this paper we rely on recent progress in 
TABLE I. Dimensionless $\left(M_{\mathrm{BH}}-\right.$ rescaled) complex frequencies of the first three QNMs for three representative values of $\chi$.

\begin{tabular}{lllll}
\hline \hline$\chi$ & $\hat{a}_{\mathrm{BH}}$ & $\sigma_{1}=\alpha_{1}+\mathrm{i} \omega_{1}$ & $\sigma_{2}=\alpha_{2}+\mathrm{i} \omega_{2}$ & $\sigma_{3}=\alpha_{3}+\mathrm{i} \omega_{3}$ \\
\hline-0.94905 & 0.37567 & $0.0871184+\mathrm{i} 0.434580$ & $0.265632+\mathrm{i} 0.415053$ & $0.455361+\mathrm{i} 0.381143$ \\
0 & 0.68703 & $0.0812684+\mathrm{i} 0.526944$ & $0.24575+\mathrm{i} 0.515117$ & $0.415019+\mathrm{i} 0.493248$ \\
+0.9695 & 0.94496 & $0.054769+\mathrm{i} 0.736715$ & $0.16459+\mathrm{i} 0.734763$ & $0.275309+\mathrm{i} 0.730986$ \\
\hline \hline
\end{tabular}

NR simulations and in particular on the free availability of hundreds of NR simulations in the Caltech-Cornell Simulating eXtreme Spacetimes (SXS) catalogue [30 32].

The approach pursued here aims at having an effective and accurate representation of the ringdown. Our approach does not aim (contrary to Refs. 27, 33 37]) at extracting the actual QNM content of NR ringdown waveforms, nor the excitation coefficients of each mode. Our work is similar in spirit to, though technically quite different from, the phenomenological ringdown model introduced in Baker et al. 38.

This paper is organized as follows. In Sec. II we briefly review the SXS data that we use. Section $\amalg$ introduces the new tool on which our analysis is based: the QNMrescaled complex ringdown waveform $\bar{h}(\tau)$. Section IV describes in detail the fitting procedure we applied to each QNM-rescaled ringdown waveform and discusses general fitting formulas that can be used outside the set of NR simulations at our disposal. Concluding remarks and outlook are collected in Sec. V. We set $G=c=1$.

\section{NUMERICAL WAVEFORM DATA}

We use sixteen waveforms produced by the CaltechCornell collaboration with the $\mathrm{S}_{\mathrm{P}} \mathrm{EC}$ code. These waveforms are publicly available through the SXS catalog 30] (these data were originally published in Refs. 26, 31, 32, 39 42]). All waveforms are equal-mass $\left(m_{1}=m_{2}\right)$, equal-spin, with the individual spins either both aligned or antialigned with the orbital angular momentum. The dimensionless individual spins are (after relaxation)

$$
\begin{aligned}
\chi \equiv \chi_{1}=\chi_{2}= & (0.9794,0.9695,0.9496,0.8997 \\
& 0.8498,0.7999,0.6000,0.43655, \\
& 0.2000,0,-0.2000,-0.43756, \\
- & 0.5999,-0.7998,-0.8996,-0.9495) .
\end{aligned}
$$

We will simply refer to them as $\chi=$ $(0.98,0.97, \pm 0.95, \pm 0.9,0.85, \pm 0.8, \pm 0.6, \pm 0.44, \pm 0.2,0)$.

We use the highest-resolution waveforms present in the catalogue, extrapolated at future null infinity using a 3rdorder polynomial ( $N=3$ label in the data). We deal here only with the asymptotic $1=m=2$ metric waveform

\footnotetext{
1 i.e., rescaled by a factor $\mathcal{R} / M$.
}

$h_{22}=A_{22} e^{-\mathrm{i} \phi_{22}}$ (with $\omega_{22}=\dot{\phi}_{22}>0$ ), and denote its $\nu$-rescaled version (with $\left.\nu=m_{1} m_{2} /\left(m_{1}+m_{2}\right)^{2}\right)$ as $h \equiv$ $h_{22} / \nu$

Figure 1 (for the high spin case $\chi=0.9695 \approx 0.97$ ) plots the complex number $h$ versus time, as a curve in a 3-dimensional space, focusing on the part of the waveform around its peak. In this paper, we define merger, occurring at $t=t_{0}$, as the peak of the modulus of $h$. Correspondingly, ringdown is defined as the signal after merger, $t>t_{0}$ : it is depicted as the thicker (red online) part of the plot.

\section{COMPLEX-NUMBER-BASED APPROACH TO QNM GENERATION}

Figure 1 highlights the complex-number nature of the ringdown signal. Here we shall show how to get a reliable effective representation of the ringdown signal $h(t)\left(t>t_{0}\right)$ by means of a multiplicative decomposition of the complex number $h(t)=h_{1}(t) \bar{h}(t)$, instead of the usual linear, additive, QNM decomposition $h(t)=h_{1}(t)+h_{2}(t)+h_{3}(t)+\cdots$. In the following we work with the dimensionless time parameter $\tau \equiv\left(t-t_{0}\right) / M_{\mathrm{BH}}$ which counts time in units of the final black hole mass $M_{\mathrm{BH}}$. The basic new idea of our approach is to factor out of $h(\tau)$ the contribution of the fundamental QNM, $h_{1}(\tau) \propto \exp \left[-\sigma_{1} \tau\right]$, where $\sigma_{1}=\alpha_{1}+\mathrm{i} \omega_{1}$ is the (dimensionless, $M_{\mathrm{BH}}$-rescaled) complex frequency of the fundamental (positive frequency, $\omega_{1}>0$ ) QNM, by defining the following QNM-rescaled ringdown waveform

$$
\bar{h}(\tau) \equiv e^{\sigma_{1} \tau+\mathrm{i} \phi_{22}^{\mathrm{mrg}}} h(\tau),
$$

where $\phi_{22}^{\mathrm{mrg}}$ is the value of $\phi_{22}$ at merger (so that $\bar{h}(\tau=$ $0)=A_{0}$ is the real amplitude of the waveform $h$ at merger). In a loose sense we can think of $\bar{h}$ as being the ringdown signal viewed in a frame rotating with the complex frequency $\omega_{1}-1 \alpha_{1}$. Fig. 2plots the parametrized curves drawn by $\bar{h}(\tau)$ in the complex plane for three values of $\chi=(-0.94905,0,+0.9695) \approx(-0.95,0,+0.97)$. The filled circle corresponds to the beginning of the ringdown, $\tau=0$. Note that the modulus of the waveform at merger is nearly independent of $\chi[16$, so that the three curves start nearly at the same point $(\bar{h}(0) \approx 1.59) \sqrt{2}$ The

2 The small variations with $\chi$ of the merger amplitude $A_{0} \equiv$ $A_{22}^{\mathrm{mrg}} / \nu$ will be quantified below. 


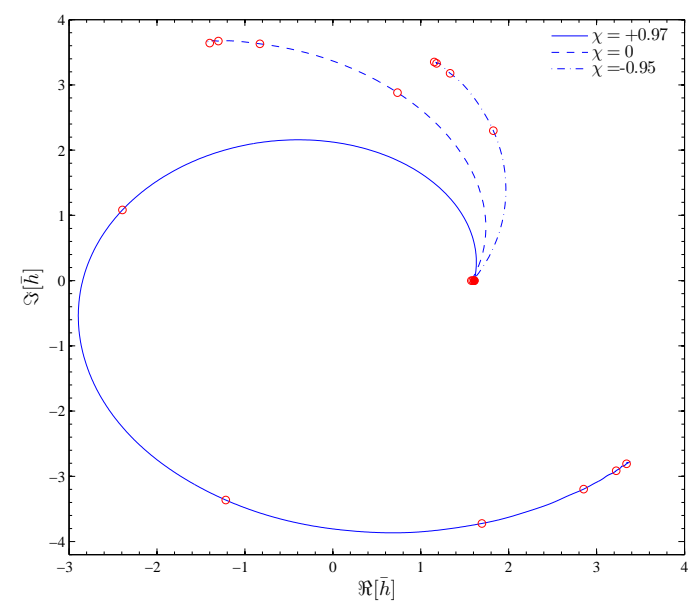

FIG. 2. Behavior of the QNM-rescaled waveform $\bar{h}$ in the complex plane for three values of $\chi$. The filled circle corresponds to merger time $\tau=0$. The empty circles mark $\Delta \tau=10$ time intervals. The time extension of each curve is $\tau_{\max }(\chi)=3.8 / \alpha_{1}(\chi)$, where $\alpha_{1}(\chi)$ is the ( $M_{\mathrm{BH}}$-rescaled) inverse damping time of the first QNM as given in Table $\square$

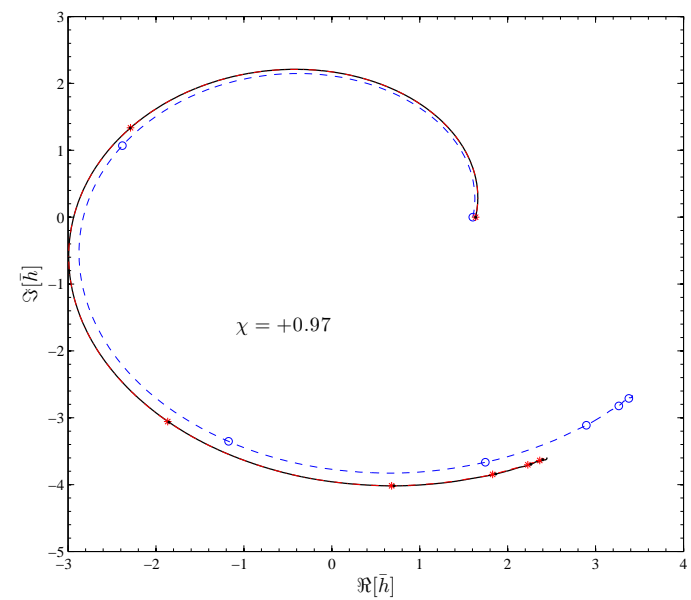

FIG. 3. The $\chi=+0.97$ complex-plane QNM-rescaled waveform $\bar{h}$ for different resolutions (either Level 5 or Level 6) and radius-extrapolations (either $N=3$ or $N=4$ ). Shown are: (i) the highest resolution (Level 6, black line with timemarkers indicated as dots) with $N=3$; (ii) the medium resolution (Level 5, red line with time-markers indicated as stars) with $N=3$; and (iii) the $N=4$ radius-extrapolation of the high-resolution $\bar{h}$ (dashed line with time-markers as circles).

other, empty, circles mark time intervals of $10 M_{\mathrm{BH}}$ after merger. We have stopped the three curves at the $\chi$-dependent time $\tau_{\max }(\chi)=3.8 / \alpha_{1}(\chi)$, corresponding to a fixed decrease in the modulus of the first QNM by a factor $\exp (-3.8) \approx 1 / 44.7$.

The striking change in the shape of these curves as $\chi$ increases from $\chi=-0.95$ to $\chi=+0.97$ is a new window on the process of QNM generation after merger. The expected analytical expression of $\bar{h}$ under the usual assumption that the QNMs are already generated just after merger would be

$$
\bar{h}_{\mathrm{QNM}}(\tau)=c_{1}+c_{2} e^{-\left(\sigma_{2}-\sigma_{1}\right) \tau}+c_{3} e^{-\left(\sigma_{3}-\sigma_{1}\right) \tau}+\cdots,
$$

with some constant, complex coefficients $c_{i}$. In Table【we list, for the three curves, the values of the dimensionless spin parameter $\hat{a}_{\mathrm{BH}}=J_{\mathrm{BH}} / M_{\mathrm{BH}}^{2}$ of the final black hole as well as the complex frequencies of the corresponding first three QNMs. Note that, while the damping coefficients $\alpha_{i}=\Re\left[\sigma_{i}\right]$ of the successive QNMs increase with QNM order (with only the first one $\alpha_{1}$ being smallish compared to 1 ), the real frequencies $\omega_{i}$ 's are nearly independent of QNM order, especially when the spin of the final black hole gets larg 3 . Therefore, the complex frequency differences $\sigma_{21} \equiv \sigma_{2}-\sigma_{1}, \sigma_{31} \equiv \sigma_{3}-\sigma_{1}$ entering Eq. (2) are approximately real and positive, especially for high spin, e.g. for $\chi=0.9695 \approx 0.97$ one has $\sigma_{21} \approx 0.109821-\mathrm{i} 0.001952 \approx 0.11$. As a consequence the curve parametrized by the mathematical expression Eq. (2) describes an approximately straight line in the complex plane of $\bar{h}$. The curve in Fig 2 corresponding to $\chi=-0.95$ is approximately straight and therefore can be well represented by a superposition of QNMs as in Eq. (2) starting at merger; i.e., for $\tau \geq 0$. The $\chi=0$ curve is still approximately straight, so that it can also be represented by a QNM sum, if one includes sufficiently many modes 4 . By contrast, the $\chi=+0.97$ case leads to a snail-shaped curve, which cannot be (easily) globally represented by a QNM sum of the type (2). We interpret this as a hint that the QNM generation is not yet completed at merger so that one would probably need to use time-dependent coefficients $c_{i}(\tau)$ in Eq. (2) to represent the ringdown signal by a (short) QNM sum. On the other hand, we see (still for $\chi=+0.97$ ) that after $\tau=20$ the $\bar{h}$ curve is approximately straight so that for $\tau \geq 20$ Eq. (2) (with constant coefficients) would allow for a reliable representation of the ringdown signal. One can say that, for $\chi=+0.97$, the generation of QNMs is completed only $\sim 20 M_{\mathrm{BH}}$ after merger. In Fig. 3 we investigate the robustness of the complex-plane $\bar{h}$ behavior under changes of resolutions and/or radius-extrapolation order. Changing the resolution has nearly no effect (apart from slightly displacing the $\Delta \tau=10$ time markers), while the effect of radius-extrapolation is more significant. In keeping with the results of Ref. [44] we use in the present work the $N=3$ extrapolated data as a compromise.

3 Reference [43] found this high-spin behavior to hold for the first 20 overtones except for the sixth one whose real frequency does not cluster with the others. The presence of such a single anomalous overtone does not significantly affect our conclusions below.

${ }^{4}$ Previous EOB works used either $N=5$ modes [17] or $N=8$ modes [15] to reliably match the ringdown waveform. 

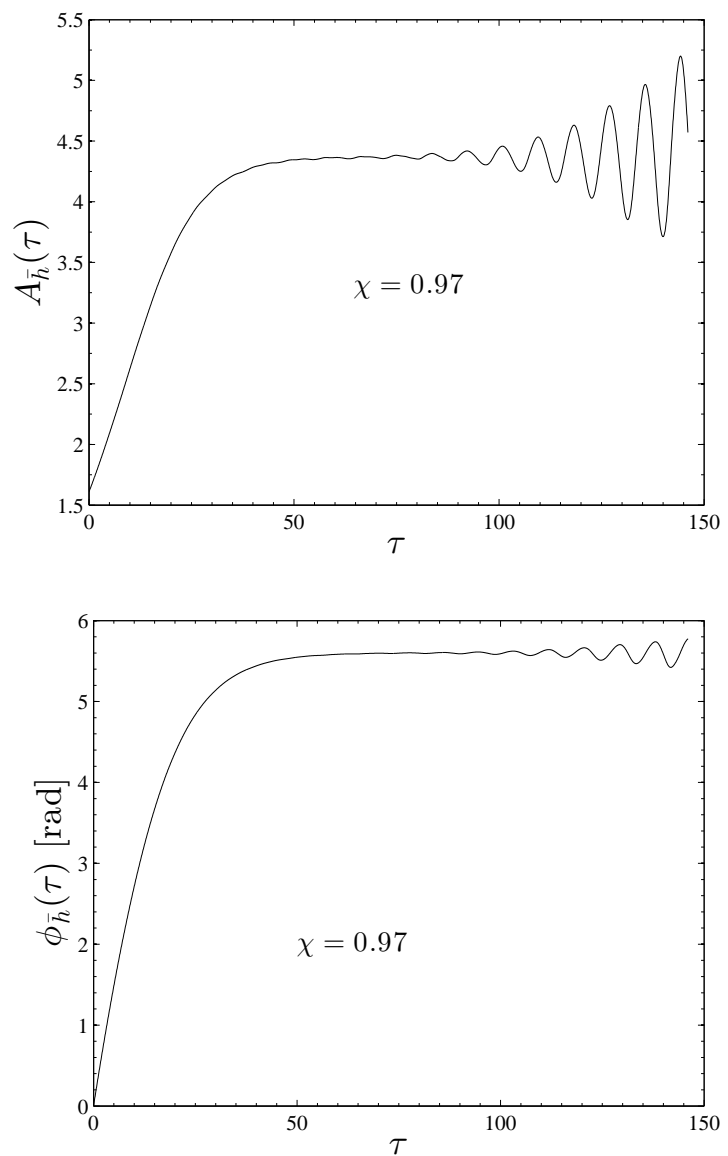

FIG. 4. Time evolution of the amplitude and phase of $\bar{h}$ for $\chi=0.97$. This plot is more extended in time than the corresponding complex-plane representation of $\bar{h}$ in Fig. 2 to highlight the amplification of numerical noise for very late times.

The complex-plane, $\bar{h}$, representation illustrated in Fig. 2 gives a new understanding of the practical need, in case one insists on representing the ringdown signal as a sum of complex frequency modes, to go beyond the actual QNM frequencies $\sigma_{i}=\alpha_{i}+\mathrm{i} \omega_{i}$ by including pseudoQNMs frequencies $\sigma_{i}^{\prime}=\alpha_{i}^{\prime}+\mathrm{i} \omega_{i}^{\prime}$ [15, 16, 18], whose role is to account for the rotation (with sizable nonzero real frequency difference, say $\left.\omega_{2}^{\prime}-\omega_{1}\right)$ we see in the $\chi=0.97$ curve. [We have checked that a similar $\bar{h}$-plane behavior explains the need for pseudo-QNM frequencies for higher multipolar waveforms, e.g. $\ell=m=4$, even in the case of low or negative $\chi$ ].

\section{AMPLITUDE-PHASE APPROACH TO RINGDOWN FITTING}

If one wanted to represent the ringdown signal as a short sum of complex frequency modes, the data behind the $\bar{h}$ curves of Fig. 2 could be used to find optimal values
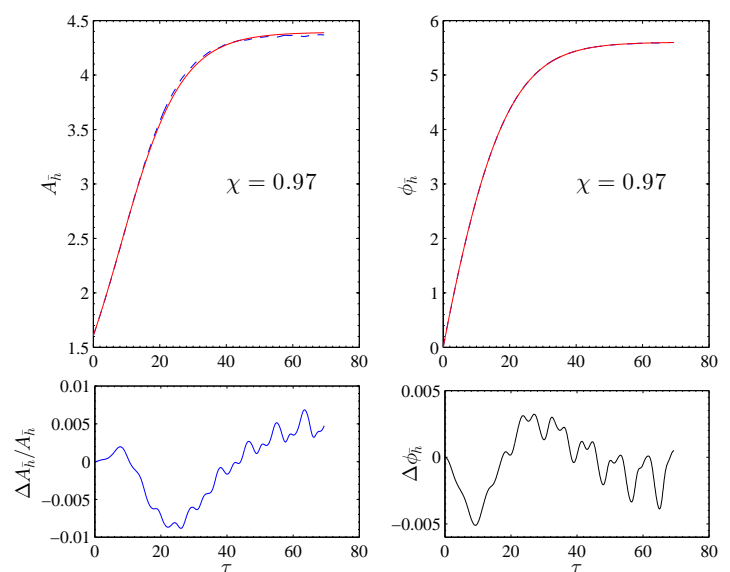

FIG. 5. Top panels: quality of the fits of $A_{\bar{h}}$ and $\phi_{\bar{h}}$ for $\chi=0.97$. The residuals are shown in the bottom panels.

TABLE II. Coefficients of the polynomial representations of: (i) the $\left(c_{3}^{A} ; c_{3}^{\phi}, c_{4}^{\phi}\right)$ coefficients entering the amplitude and phase fitting templates (4)-(5), and (ii) the QNM/NR data entering the osculation constraints Eqs. (6)-(10), with $\Delta \omega \equiv \omega_{1}-M_{\mathrm{BH}} \omega_{22}^{\mathrm{mrg}}$.

\begin{tabular}{llllll}
\hline \hline & $p_{4}$ & $p_{3}$ & $p_{2}$ & $p_{1}$ & $p_{0}$ \\
\hline$c_{3}^{A}$ & 0 & 0 & 0.044763 & -0.138980 & -0.366538 \\
$c_{3}^{\phi}$ & -1.174263 & -0.9099211 & 1.690678 & 2.866629 & 4.188784 \\
$c_{4}^{\phi}$ & 0 & 0 & 2.925663 & 4.362706 & 2.462696 \\
\hline$\hat{A}_{22}^{\text {mrg }}$ & 0.014175 & 0.014553 & 0.012896 & -0.004458 & 1.575613 \\
$\alpha_{21}$ & -0.009068 & -0.013719 & -0.012981 & -0.022385 & 0.164398 \\
$\alpha_{1}$ & -0.004416 & -0.006810 & -0.006789 & -0.010196 & 0.081224 \\
$\Delta \omega$ & 0.020975 & 0.028444 & 0.026957 & 0.066588 & 0.184738 \\
\hline \hline
\end{tabular}

of any additional pseudo-QNM frequency $\sigma_{i}^{\prime}$. However, we wish here to suggest an alternative strategy for analytically representing the ringdown signal. The QNMrescaled ringdown waveform $\bar{h}(\tau) \equiv e^{\sigma_{1} \tau+\mathrm{i} \phi_{22}^{\mathrm{mrg}}} h(\tau)$ being a complex quantity, can be decomposed in amplitude and phase

$$
\bar{h}(\tau) \equiv A_{\bar{h}}(\tau) e^{+\mathrm{i} \phi_{\bar{h}}(\tau)}
$$

[Note the sign convention: $\phi_{\bar{h}}(\tau)=\omega_{1} \tau-\phi_{22}(\tau)+\phi_{22}^{\mathrm{mrg}}$ ]. Figure 4 exhibits the time evolution (after merger $\tau \geq 0$ ) of the amplitude and phase $\left(A_{\bar{h}}, \phi_{\bar{h}}\right)$ of $\bar{h}$ for $\chi=0.97$. The shapes of the curves $A_{\bar{h}}(\tau)$ and $\phi_{\bar{h}}(\tau)$ are similar and are both reminiscent of a hyperbolic tangent. Other values of $\chi$ lead to very similar shapes. The plateau behavior of the curves as $\tau$ increases is linked to the asymptotic behavior $\bar{h}(\tau) \approx \bar{h}_{\mathrm{QNM}}(\tau) \sim c_{1}+\mathcal{O}\left(e^{-\left(\alpha_{2}-\alpha_{1}\right) \tau}\right)$ given by Eq. (2). [The oscillations around the plateau that one sees on these curves for $\tau \gtrsim 80$ are due to the amplification of numerical noise by the exponentially growing 

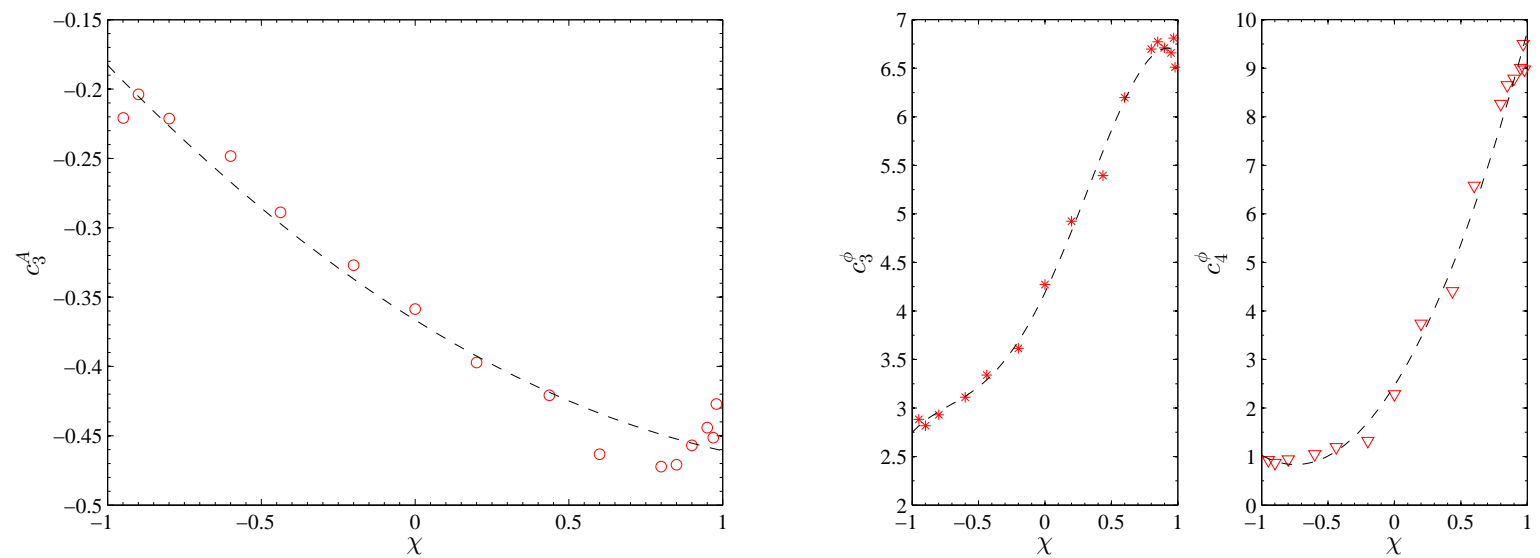

FIG. 6. Behavior of the six fitting coefficients versus dimensionless spin $\chi$. The numerical points can be well fitted by simple polynomials in $\chi$. The corresponding coefficients are listed in Table II

factor $\left.e^{\sigma_{1} \tau}\right]$. We found that, at an effective level, $A_{\bar{h}}(\tau)$ and $\phi_{\bar{h}}(\tau)$ can be accurately represented by the following general functional forms

$$
\begin{aligned}
& A_{\bar{h}}(\tau)=c_{1}^{A} \tanh \left(c_{2}^{A} \tau+c_{3}^{A}\right)+c_{4}^{A}, \\
& \phi_{\bar{h}}(\tau)=-c_{1}^{\phi} \ln \left(\frac{1+c_{3}^{\phi} e^{-c_{2}^{\phi} \tau}+c_{4}^{\phi} e^{-2 c_{2}^{\phi} \tau}}{1+c_{3}^{\phi}+c_{4}^{\phi}}\right) .
\end{aligned}
$$

[Note that when $c_{4}^{\phi}=0$, the time-derivative of $\phi_{\bar{h}}(\tau)$ differs by a constant from a hyperbolic tangent]. In addition, we constrain the parameters entering these functional forms by imposing simple physical constraints similar to the ones imposed in the usual linear QNM representation of the ringdown. Namely, we impose: (i) that the value of $A_{\bar{h}}(\tau)$ at $\tau=0$ coincide with the NR amplitude at merger $\hat{A}_{22}^{\mathrm{mrg}} \equiv A_{22}^{\mathrm{mrg}} / \nu$; (ii) the value of $d A_{\bar{h}}(\tau) / d \tau$ at $\tau=0$ so that the $\dot{A}_{22}=0$ at merger; (iii) that the amplitude exponential-decay parameter $c_{2}^{A}$ coincide with $\alpha_{21} / 2$, where $\alpha_{21} \equiv \alpha_{2}-\alpha_{1}$; (iv) that $d \phi_{\bar{h}}(\tau) / d \tau$ at $\tau=0$ be equal to $\Delta \omega \equiv \omega_{1}-M_{\mathrm{BH}} \omega_{22}^{\mathrm{mrg}}$; and $(\mathrm{v})$ that the phase exponential-decay parameter $c_{2}^{\phi}$ be equal to $\alpha_{21}$. Imposing these five constraints allow one to keep only one free parameter, namely $c_{3}^{A}$, in the amplitude template Eq. (4), and two free parameters, namely $c_{3}^{\phi}$ and $c_{4}^{\phi}$, in the phasing template, Eq. (5). Specifically, the other parameters are expressed in terms of these three independent parameters as follows

$$
\begin{aligned}
c_{2}^{A} & =\frac{1}{2} \alpha_{21}, \\
c_{1}^{A} & =\hat{A}_{22}^{\mathrm{mrg}} \alpha_{1} \frac{\cosh ^{2} c_{3}^{A}}{c_{2}^{A}}, \\
c_{4}^{A} & =\hat{A}_{22}^{\mathrm{mrg}}-c_{1}^{A} \tanh c_{3}^{A}, \\
c_{2}^{\phi} & =\alpha_{21}, \\
c_{1}^{\phi} & =\frac{1+c_{3}^{\phi}+c_{4}^{\phi}}{c_{2}^{\phi}\left(c_{3}^{\phi}+2 c_{4}^{\phi}\right)}\left(\omega_{1}-M_{\mathrm{BH}} \omega_{22}^{\mathrm{mrg}}\right) .
\end{aligned}
$$

In the present paper, we propose as effective strategy for analytically representing the ringdown to least-square fit the QNM-rescaled NR ringdown waveform, $\bar{h}_{\mathrm{NR}}(\tau)$, to the templates (4), (15), constrained by Eqs. (6)-(10), so as to determine best-fit values of the three coefficients $c_{3}^{A}$, $c_{3}^{\phi}$ and $c_{4}^{\phi}$. We have chosen as $\tau$ interval for the fitting $0 \leq \tau \leq 3.8 / \alpha_{1}(\chi)$. Indeed $\tau_{\max }=3.8 / \alpha_{1}(\chi)$ happens to be well on the plateau while still avoiding the region where the oscillations get significant (e.g., $\tau_{\max } \approx 69.38$ for $\chi=0.97)$. The quality of the fit performance is illustrated in Fig. 5 for $\chi=0.97$. Note in particular how the phasing is reproduced within $5 \times 10^{-3}$ radians. In a first version of this analysis we used the same (but unconstrained) amplitude template and the following simpler, two-parameter, unconstrained phasing template $\phi_{\bar{h}}(\tau)=c_{1}^{\phi} \tanh \left(c_{2}^{\phi} \tau\right)$. Such choices led to a comparably accurate representation of the ringdown for $\tau \gtrsim 10$, but to larger disagreements for $0 \leq \tau \lesssim 10$.

We have applied this strategy to the sixteen waveforms of the SXS catalog. For each waveform, i.e. for each $\chi$, the ringdown information is encoded in the set of three coefficients $\left(c_{3}^{A} ; c_{3}^{\phi}, c_{4}^{\phi}\right)$. We have found that the $\chi$-dependence of the $c_{i}^{(A, \phi)}$, s is relatively smooth (see Fig. 6). One can approximately represent these coefficients as second-order polynomials in $\chi$ except for $c_{3}^{\phi}$ for 

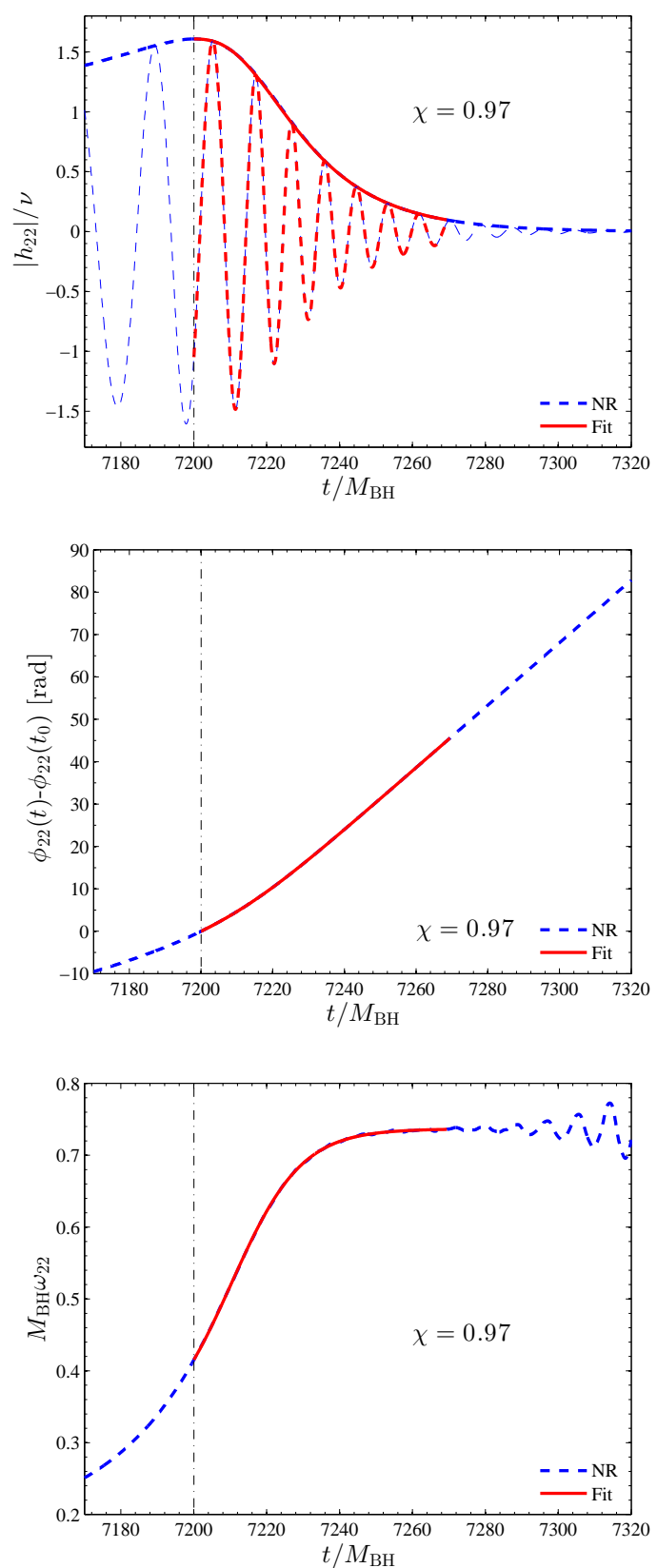

FIG. 7. NR waveform (dashed line) and its ringdown fit (solid line, for $0 \leq \tau \leq 3.8 / \alpha_{1}(\chi)$ ) for $\chi=0.97$ : amplitude (top panel), phase (middle panel) and frequency (bottom panel). The vertical lines correspond to the merger, defined as the peak of $\left|h_{22}\right| / \nu$.

which we found that a fourth-order polynomial gives a better fit5. To complete the information needed to use our results we also provide fits for the $\chi$-dependence of

\footnotetext{
${ }^{5}$ For completeness, let us mention that a (less accurate) secondorder fit for $c_{3}^{\phi}$ reads $c_{3}^{\phi}=0.479448 \chi^{2}+2.176818 \chi+4.342270$.
}

$\alpha_{1}, \alpha_{21}, \hat{A}_{22}^{\mathrm{mrg}}$ and $\Delta \omega \equiv \omega_{1}-M_{\mathrm{BH}} \omega_{22}^{\mathrm{mrg}}$. All our fits are done with the convention $c_{i}(\chi)=p_{4} \chi^{4}+p_{3} \chi^{3}+p_{2} \chi^{2}+$ $p_{1} \chi+p_{0}$. The explicit values of the $p_{n}$ coefficients are listed in Table [I]

The comparison between the $\chi$-fits for $\left(c_{3}^{A} ; c_{3}^{\phi}, c_{4}^{\phi}\right)$ and the raw points is displayed in Fig. 6. The amplitude coefficient plot shows more scatter around the fit, probably due to amplified numerical noise (this is consistent with the fact that the oscillation around the plateau is larger for amplitude than for phase, see Fig. 4). We have checked that by reducing the $\tau$-length of the fitting interval the scatter could be reduced, especially for large spins. Changing the extrapolation order $N=3$ to $N=2$ reduces the oscillations around the plateaux in Fig. 4 and thereby the scatter. We have checked that inserting the $\chi$-fitted versions of $\left(c_{3}^{A} ; c_{3}^{\phi}, c_{4}^{\phi}\right)$ and of $\left(\hat{A}_{22}^{\mathrm{mrg}}, \alpha_{21}, \alpha_{1}, \Delta \omega\right)$ in our functional forms Eq. (4)-(10) leads to representations of the ringdown with phase and fractional amplitude disagreements that remain smaller than about 0.04 in all cases.

Finally, the very satisfactory representation, given by our strategy, of the original ringdown waveform $h(t)=$ $h_{22} / \nu$ (decomposed in amplitude, phase and frequency), is displayed in Fig. 7 for the case $\chi=0.97$. The corresponding phase and fractional amplitude differences were given in the bottom panels of Fig. 5 .

\section{CONCLUSIONS AND OUTLOOK}

Let us summarize our main results.

1. We introduced a new tool for analyzing ringdown waveforms, consisting of studying the time evolution, after merger, of the QNM-rescaled complex quantity $\bar{h}(\tau)$, Eq. (1).

2. Using publicly available SXS, Caltech-Cornell, waveform data 30] spanning dimensionless spins $-0.95 \leq \chi \leq 0.98$, and the $\bar{h}$ tool, we have found that, in the case of large spin, the ringdown signal is compatible with the usually expected sum, Eq. (2), of Kerr black hole quasi-normal-modes complex frequencies, only for times sufficiently posterior to merger, e.g., by about $20 M_{\mathrm{BH}}$ when $\chi=0.97$. For earlier times the $\bar{h}$ diagnostics can be seen as a new tool to study the building up of QNMs just after merger.

3. To get an analytic representation of the ringdown signal starting just after merger we emphasized that two strategies are possible: (i) to introduce, as in Refs. [15, 16, 18], pseudo-QNM frequencies, in which case our $\bar{h}$ diagnostics can provide an efficient tool for optimizing their determination; or (ii) to separately fit the amplitude and phase of the QNM-rescaled signal $\bar{h}(\tau)$ by hyperbolic-tangentbased templates. 
4. We have applied the latter strategy to sixteen, equal-mass, equal-spin, $\ell=m=2$ waveforms of the SXS catalog and showed that it leads to a very accurate representation of the ringdown waveform (with phase differences comparable to numerical errors). We have checked that our strategy also yields accurate representations of ringdown waveforms for unequal mass, spin-aligned SXS waveforms.

5. We provided explicit polynomial representations as functions of $\chi$ of the coefficients entering our fitting templates for the amplitude and phase of the ringdown signal.

6. The quality of our fits suggests that our method will give reliable representations of the ringdown waveforms also for values of the spin not included in the SXS catalog. The method allows one to interpolate between the catalogued $\chi$ values, and hopefully also to extrapolate the full ringdown waveform to more extremal spin values. For example, we can predict the energy radiated during the ringdown versus spin.

Our findings open the following avenues for further research.

(a) The extension of our approach to higher multipolar modes $(\ell>2)$ is conceptually straightforward, but should be quantified. In particular, it will be interesting to investigate to what extent the behavior of higher multipolar modes during ringdown is not representable [because of an excessive rotation of $\bar{h}(\tau)$ ] as a sum of QNMs with constant coefficients.

(b) A preliminary investigation of the behavior of $\bar{h}(\tau)$ in the ringdown waveform generated by a point particle inspiralling and plunging on a (fastspinning) Kerr black hole has shown that the rotating features of $\bar{h}(\tau)$ are even more marked in that case [45]. Future work on this case will hopefully improve our knowledge of the generation mechanism of QNMs.

(c) The optimal choice of extrapolation order for the numerical waveform must be further investi- gated. Similarly, it will be interesting to analyze also different NR waveform data obtained with independent infrastructures, so as to gauge possible (tiny) systematics present in extrapolated SXS data.

(d) The quality of our fits should be quantified, using data-analysis-relevant measures. This might allow one to use variations on our fits such as: (i) deleting some of our constraints; (ii) modifying them6; or (iii) adding further constraints so as to impose a higher order osculation of frequency and amplitude at merger. For instance, we found that relaxing the phase constraints Eqs. (9)-(10) leads to an even better phasing agreement with a very flat behavior (within $\pm 10^{-3} \mathrm{rad}$ ) of $\Delta \phi_{\bar{h}}$ as a function of $\tau$.

(e) The fitting procedure presented here could be systematically applied to all waveforms present in the SXS catalog, starting from unequal-mass, but spin aligned configurations. Its generalization to non-spin-aligned binaries should be explored. Including also higher multipoles and small-mass-ratio waveforms computed from perturbative calculations (solving either the Regge-Wheeler-Zerilli or Teukolsky equations) the procedure outlined here might give an efficient representation of the complete ringdown waveform as a function of the spin magnitude and mass ratio.

(f) Finally, technical ways of using the dynamics of the QNM-rescaled waveform $\bar{h}(\tau)$ for finding optimal values of additional pseudo-QNM complex frequencies could be explored and compared to the result of the new strategy that we have proposed here.

\section{ACKNOWLEDGMENTS}

We thank Andrea Taracchini for useful comments.
[1] A. Buonanno and T. Damour, Phys. Rev. D59, 084006 (1999).

[2] A. Buonanno and T. Damour, Phys. Rev. D62, 064015 (2000).

${ }^{6}$ For instance by replacing the second exponential $e^{-2 c_{2}^{\phi} \tau}$ in Eq. (5) by $e^{-\left(\alpha_{3}-\alpha_{1}\right) \tau}$.
[3] T. Damour, P. Jaranowski, and G. Schaefer, Phys. Rev. D62, 084011 (2000).

[4] T. Damour, Phys. Rev. D64, 124013 (2001).

[5] A. Buonanno, Y. Chen, and T. Damour, Phys. Rev. D74, 104005 (2006), gr-qc/0508067.

[6] T. Damour, B. R. Iyer, and A. Nagar, Phys. Rev. D79, 064004 (2009).

[7] T. Damour and A. Nagar, Phys.Rev. D81, 084016 (2010), 0911.5041. 
[8] A. Buonanno, Y. Pan, J. G. Baker, J. Centrella, B. J. Kelly, et al., Phys.Rev. D76, 104049 (2007), 0706.3732.

[9] T. Damour and A. Nagar, Phys.Rev. D77, 024043 (2008), 0711.2628.

[10] T. Damour, A. Nagar, E. N. Dorband, D. Pollney, and L. Rezzolla, Phys.Rev. D77, 084017 (2008), 0712.3003.

[11] T. Damour, A. Nagar, M. Hannam, S. Husa, and B. Bruegmann, Phys.Rev. D78, 044039 (2008), 0803.3162.

[12] M. Boyle, A. Buonanno, L. E. Kidder, A. H. Mroue, Y. Pan, et al., Phys.Rev. D78, 104020 (2008), 0804.4184.

[13] A. Buonanno, Y. Pan, H. P. Pfeiffer, M. A. Scheel, L. T. Buchman, et al., Phys.Rev. D79, 124028 (2009), 0902.0790

[14] T. Damour, A. Nagar, D. Pollney, and C. Reisswig, Phys.Rev.Lett. 108, 131101 (2012), 1110.2938.

[15] Y. Pan, A. Buonanno, M. Boyle, L. T. Buchman, L. E. Kidder, et al., Phys.Rev. D84, 124052 (2011), 1106.1021.

[16] A. Taracchini, Y. Pan, A. Buonanno, E. Barausse, M. Boyle, et al., Phys.Rev. D86, 024011 (2012), 1202.0790

[17] T. Damour, A. Nagar, and S. Bernuzzi, Phys.Rev. D87, 084035 (2013), 1212.4357.

[18] A. Taracchini, A. Buonanno, Y. Pan, T. Hinderer, M. Boyle, et al., Phys.Rev. D89, 061502 (2014), 1311.2544

[19] Y. Pan, A. Buonanno, A. Taracchini, M. Boyle, L. E. Kidder, A. H. Mroué, H. P. Pfeiffer, M. A. Scheel, B. Szilágyi, and A. Zenginoglu, Phys. Rev. D 89, 061501 (2014).

[20] Y. Pan, A. Buonanno, A. Taracchini, L. E. Kidder, A. H. Mroué, H. P. Pfeiffer, M. A. Scheel, and B. Szilágyi, Phys. Rev. D 89, 084006 (2014).

[21] T. Damour, A. Nagar, and L. Villain, Phys.Rev. D89, 024031 (2014), 1307.2868.

[22] T. Damour, F. Guercilena, I. Hinder, S. Hopper, A. Nagar, and L. Rezzolla, Phys. Rev. D 89, 081503 (2014).

[23] T. Damour and A. Nagar, Phys. Rev. D76, 064028 (2007), 0705.2519.

[24] Y. Pan, A. Buonanno, R. Fujita, E. Racine, and H. Tagoshi, Phys.Rev. D83, 064003 (2011), 1006.0431.

[25] L. Blanchet, Living Rev.Rel. 17, 2 (2014), 1310.1528.
[26] D. A. Hemberger, G. Lovelace, T. J. Loredo, L. E. Kidder, M. A. Scheel, et al., Phys.Rev. D88, 064014 (2013), 1305.5991.

[27] L. London, J. Healy, and D. Shoemaker (2014), 1404.3197

[28] E. Berti, V. Cardoso, and C. M. Will, Phys.Rev. D73, 064030 (2006), gr-qc/0512160.

[29] E. Berti, http://www.phy.olemiss.edu/berti/qnms.html.

[30] http://www.black-holes.org/waveforms

[31] A. H. Mroue, M. A. Scheel, B. Szilagyi, H. P. Pfeiffer, M. Boyle, et al., Phys.Rev.Lett. 111, 241104 (2013) 1304.6077.

[32] G. Lovelace, M. Scheel, and B. Szilagyi, Phys.Rev. D83, 024010 (2011), 1010.2777.

[33] E. Berti, V. Cardoso, J. A. Gonzalez, U. Sperhake, M. Hannam, et al., Phys.Rev. D76, 064034 (2007), grqc/0703053.

[34] E. Berti, J. Cardoso, V. Cardoso, and M. Cavaglia, Phys.Rev. D76, 104044 (2007), 0707.1202.

[35] E. Berti, V. Cardoso, J. A. Gonzalez, and U. Sperhake, Phys.Rev. D75, 124017 (2007), gr-qc/0701086.

[36] S. Hadar, B. Kol, E. Berti, and V. Cardoso, Phys.Rev. D84, 047501 (2011), 1105.3861.

[37] A. Taracchini, A. Buonanno, G. Khanna, and S. A. Hughes (2014), 1404.1819.

[38] J. G. Baker, W. D. Boggs, J. Centrella, B. J. Kelly, S. T. McWilliams, et al., Phys.Rev. D78, 044046 (2008), 0805.1428 .

[39] T. Chu, H. P. Pfeiffer, and M. A. Scheel, Phys.Rev. D80, 124051 (2009), 0909.1313.

[40] G. Lovelace, M. Boyle, M. A. Scheel, and B. Szilagyi, Class.Quant.Grav. 29, 045003 (2012), 1110.2229.

[41] L. T. Buchman, H. P. Pfeiffer, M. A. Scheel, and B. Szilagyi, Phys.Rev. D86, 084033 (2012).

[42] A. H. Mroue and H. P. Pfeiffer (2012), 1210.2958.

[43] H. Onozawa, Phys.Rev. D55, 3593 (1997), grqc/9610048.

[44] M. Boyle and A. H. Mroue, Phys. Rev. D80, 124045 (2009), 0905.3177.

[45] S. Bernuzzi, T. Damour, E. Harms, and A. Nagar (2014), in preparation. 